Revue de droit comparé du travail et de la sécurité sociale

2| 2018

Harcèlement moral au travail en jurisprudence

comparée

\title{
Le harcèlement moral au travail dans la jurisprudence chilienne
}

Pablo Arellano Ortiz et Juan Pablo Severin Concha

(2) OpenEdition

Journals

Édition électronique

URL : https://journals.openedition.org/rdctss/1946

DOI : $10.4000 /$ rdctss. 1946

ISSN : 2262-9815

Éditeur

Centre de droit comparé du travail et de la sécurité sociale

Édition imprimée

Date de publication : 1 juin 2018

Pagination : 50-57

ISSN : 2117-4350

Référence électronique

Pablo Arellano Ortiz et Juan Pablo Severin Concha, « Le harcèlement moral au travail dans la jurisprudence chilienne », Revue de droit comparé du travail et de la sécurité sociale [En ligne], 2 | 2018, mis en ligne le 01 novembre 2021, consulté le 12 novembre 2021. URL : http:// journals.openedition.org/rdctss/1946 ; DOI : https://doi.org/10.4000/rdctss.1946

\section{(c) (i) $\ominus$}

Revue de droit comparé du travail et de la sécurité sociale est mise à disposition selon les termes de la Licence Creative Commons Attribution - Pas d'Utilisation Commerciale - Pas de Modification 4.0 International. 


\section{LE HARCÈLEMENT MORAL AU TRAVAIL DANS LA JURISPRUDENCE CHILIENNE}

\section{Pablo Arellano O ${ }^{1}$}

Spécialiste du Droit du travail, Unité de droit du travail et de la réforme,

Département de la Gouvernance et du Tripartisme de l'OIT, Genève;

Chercheur à la Faculté de droit, Université pontificale catholique de Valparaiso

\section{Juan Pablo Severin C}

Professeur de Droit du travail, Université catholique du nord, Coquimbo

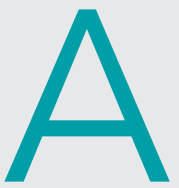

u Chili, deux périodes se sont succédées concernant la protection des salariés exposés à des situations de harcèlement au travail. Durant une première phase, ce phénomène n'était pas envisagé par le droit du travail et la protection en cas de harcèlement au travail trouve son origine dans une jurisprudence fondée sur l'obligation de l'employeur de prendre toutes les mesures nécessaires pour protéger efficacement la vie et la santé des salariés et sur la considération que les comportements de harcèlement au travail impliquaient une violation des droits fondamentaux des salariés. La seconde étape débute par la reconnaissance légale expresse du harcèlement au travail dans le Code du travail chilien, grâce à la loi n² 20.607 du 8 août $2012^{2}$.

1 Les opinions exprimées sont celles de l'auteur et ne représentent pas nécessairement celles de l'Organisation internationale du travail.

2 Pour en savoir plus sur cette loi, il est conseillé de consulter E. Caamaño Rojo, J.L. Ugarte Cataldo, "El acoso laboral: tutela y prueba de la lesión de los derechos fundamentales », Revista lus et Praxis, Université de Talca - Faculté des Sciences juridiques et sociales, $\mathrm{n}^{\circ} 1$ 2014, p. 67-90 ; K. Lippel, y L.Casas Becerra„ " Derecho y acoso desde la perspectiva comparada ", Estudios Laborales $n^{\circ} 7$, revue de la Sociedad Chilena de Derecho del Trabajo y de la Seguridad Social, Legal Publishing, 2012, p. 5-24 ; L. Casas Becerra, "Un estudio sobre las quejas por acoso sexual ante la inspección del Trabajo: Desafíos y oportunidades de la ley $n^{\circ} 20.607$ ", Estudios Laborales, n 7, Revue de la Sociedad Chilena de Derecho del Trabajo y de la Seguridad Social, Legal Publishing, 2012, p. 25-50. E. Caamaño Rojo," "La tipificación y sanción del acoso laboral en Chile: Un ejemplo de cómo retroceder en el resguardo de los derechos fundamentales ", Estudios Laborales $n^{\circ} 7$, revue de la Sociedad Chilena de Derecho del Trabajo y de la Seguridad Social, Legal Publishing, 2012, p. 179-202 ; E. Caamaño Rojo, "Propuestas para la delimitación de la noción jurídico-laboral del acoso moral o mobbing », Estudios Laborale, $s n^{\circ} 6$, revue de la Sociedad Chilena de Derecho del Trabajo y de la Seguridad Social, Legal Publishing, 2012, p. 71-92 ; F. Walker Errazuriz, y P. Arellano Ortiz, Derecho de las relaciones laborales, Tomo 1 Derecho individual del trabajo, Librotecnia, Santiago, 2016. 
Dans le domaine des relations individuelles de travail, le Code du travail est applicable à la majorité des salariés. II ne s'applique pas aux travailleurs de la fonction publique étatique, qu'ils soient centralisés ou décentralisés, du Congrès national et du Pouvoir judiciaire, ni aux salariés des entreprises ou institutions de l'État ou de celles dans lesquelles l'État a des contributions, une participation ou une représentation, à condition que ces salariés soient soumis à une loi ou à un statut spécial. Toutefois, les salariés des entités susmentionnées sont soumis aux règles du Code du travail sur des aspects ou des questions qui ne sont pas régis par leurs statuts respectifs, à condition qu'elles ne soient pas contraires à ces derniers. Cette application supplétive de la législation générale du travail aux fonctionnaires s'est considérablement renforcée ces dernières années, et l'on observe une tendance à soumettre la fonction publique au Code du travail. Cette tendance n'est pas sans rapport avec la règlementation sur le harcèlement au travail inscrite dans le Code du travail, qui s'appliquera aux fonctionnaires des services publics et des municipalités.

Dans la première partie de cet article, nous traiterons de la réglementation du harcèlement au travail, en faisant état de la situation précédant l'adoption de la loi, puis nous détaillerons la loi qui le régit expressément(I). En outre, nous examinerons brièvement comment les normes de protection du harcèlement au travail ont été interprétées et appliquées par les tribunaux chiliens (II).

\section{I - LA RÉGLEMENTATION DU HARCÈLEMENT AU TRAVAIL}

Comme nous l'avons déjà mentionné, avant que le harcèlement au travail ne soit expressément régi par la loi $n^{\circ} 20.607$, la garantie de la dignité et de l'intégrité des salariés leur permettait d'être protégés contre un tel comportement. Pour sanctionner ces comportements de harcèlement, les tribunaux chiliens, à l'occasion de procédures judicaires devant les tribunaux du travail appliquaient la règle énoncée dans la première partie de l'article 2, paragraphe 2, du Code du travail, qui dispose que « les relations de travail doivent toujours être fondées sur un traitement compatible avec la dignité de la personne ", interprétée à la lumière de l'article 184, paragraphe 1, du Code du travail, qui dispose que l'employeur « est tenu de prendre toutes les mesures nécessaires pour protéger efficacement la vie et la santé des salariés... ».

Depuis l'introduction d'une nouvelle procédure de protection des droits fondamentaux des salariés, tout comportement de harcèlement au travail qui viole les droits à l'intégrité physique et psychologique, à l'honneur ou à la vie privée, doit faire l'objet d'un recours auprès du tribunal du travail, compétent pour statuer sur de telles demandes. Ainsi, avant même que le Code du travail ne règlemente le harcèlement au travail, les tribunaux ont assuré la protection de ceux qui étaient victimes d'un tel comportement ${ }^{3}$.

3 Pour de plus amples développements sur la jurisprudence relative à la protection en cas de harcèlement au travail, il convient de se rapporter à S. Gamonal Contreras, P. Prado López, El

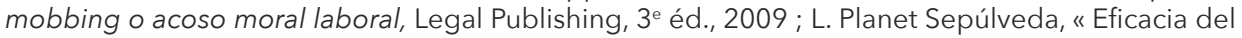
procedimiento de tutela laboral como protección contra el mobbing en Chile », Revista Chilena de Derecho de Trabajo y de la Seguridad Social, Département du Droit du travail et de la Sécurité sociale, Faculté de Droit, Université du Chili, vol 1, n¹, 2010, p. 93-122 ; M.C. Irigoyen Peduzzi, «El Acoso Moral », Revista Laboral Chilena, 2010, n 11, p. 63. E. Caamaño Rojo, "La noción de acoso moral laboral o mobbing y su reconocimiento por la jurisprudencia en Chile ", Revista de Derecho de la Université pontificale catholique de Valparaíso, 2011, n³7, p. 215-240. 
La loi n²0.607 précise à l'article 2 du Code du travail que le harcèlement au travail " est contraire à la dignité de la personne » et le définit comme « tout comportement qui constitue une agression ou un harcèlement répété de la part de l'employeur ou d'un ou plusieurs salariés, contre un ou plusieurs autres salariés, par quelque moyen que ce soit, ayant pour conséquence de dévaloriser, maltraiter ou humilier la ou les victimes, ou qui menace ou nuit à leur carrière ou à leur avenir professionnel».

La Direction du travail a souligné, qu'à partir du texte sur le harcèlement au travail introduit au deuxième paragraphe de l'article 2 du Code du travail, il est possible de déduire « que l'intention du législateur était, d'une part, de sanctionner le harcèlement au travail, survenu dans les conditions prévues par la nouvelle législation, comme étant contraire à la dignité de la personne, puisqu'il s'agit d'un comportement illégal qui porte atteinte à diverses prérogatives juridiques découlant de ce droit, telles que l'intégrité physique et mentale et l'égalité des chances, ayant ainsi pour fondement le respect et la promotion des droits fondamentaux des individus dans le cadre de leur travail, protégés par la Constitution et les lois, dont les plus importantes sont directement applicables, notamment celle du Titre III du Livre I du Code du travail, du Règlement intérieur qui, dans son article 153, paragraphe 2, prescrit : «Il convient notamment de fixer les règles à respecter pour garantir un environnement de travail décent et propice au respect entre les salariés $»^{4}$. La Direction du travail réaffirme également l'importance des règles existantes dans le Code du travail concernant l'obligation de l'employeur de protéger le salarié, en particulier l'article 184.

La réforme législative qui a introduit une définition du harcèlement au travail contient une terminologie qui pourrait donner lieu à des problèmes d'interprétation. La Direction du travail, le prévoyant, a précisé, dans son avis du 9 août $2012^{5}$, le sens de ses principales expressions, en déclarant ce qui suit :

- Agression : "acte d'attaquer quelqu'un pour le tuer, le blesser ou lui faire mal ». L'agression se définit également comme un « acte contraire au droit d'un tiers».

- Intimidation : " action et effet d'intimider » et parmi les acceptions de son infinitif, figurent: «Vexer quelqu'un ou se moquer de lui avec insistance » et «Inciter quelqu'un avec insistance pour qu'il fasse quelque chose».

- Dévalorisation : "effet de dévaloriser ", l'infinitif « dévaloriser » a aussi plusieurs acceptions, notamment « Dénigrer ou porter atteinte à l'honneur ou la réputation ».

- Maltraitance : «action et effet de maltraiter», son infinitif «maltraiter » est défini comme «maltraiter quelqu'un par la parole ou par l'action » et aussi « dévaloriser, pervertir ».

- Humiliation: " action et effet d'humilier ou de s'humilier, et l'infinitif "humilier", quant à lui, est défini comme " atteindre quelqu'un dans son amour-propre, sa dignité » et "Pour une personne : vivre une situation dans laquelle sa dignité est rabaissée ».

Sur la base de ces définitions, la Direction du travail conclut que «le législateur a envisagé au sens large les comportements considérés comme du harcèlement au travail, de manière à permettre de considérer comme tel tout comportement qui implique une

4 Avis de la Direction du travail 3519/034 du 9 août 2012, qui établit le sens et la portée des modifications apportées par la loi n²0.607 au texte du deuxième paragraphe de l'article 2, du premier paragraphe de l'article 160, et du deuxième et sixième paragraphe de l'article 171, tous issus du Code du travail.

5 lbid. 
agression physique contre le ou les salariés touchés, ainsi que les moqueries ou ennuis insistants à leur encontre, l'incitation à accomplir quelque chose, à condition que tout comportement de ce genre soit répété, et ce, quels que soient les moyens par lesquels les victimes sont soumises aux agressions ou aux harcèlements $»^{6}$.

Concernant l'expression « ou qui menace ou nuit à leur carrière ou à leur avenir professionnel » qui figure dans la définition légale, cela doit être compris en ce sens que «le harcèlement au travail se produit non seulement lorsque l'action de l'employeur ou du/des salarié(s) cause un préjudice professionnel direct à leur situation au sein de l'entreprise, mais aussi lorsque la création d'un environnement de travail hostile et agressif met leur évolution professionnelle en danger ${ }^{7}$.

En d'autres termes, le harcèlement en milieu de travail doit être entendu comme un comportement persistant et non comme des actes isolés. Un tel comportement doit être d'une ampleur telle qu'il a des effets sur la personne ou qu'il menace sa situation de travail ou son emploi.

Non seulement la loi $n^{\circ} 20.607$ décrit le harcèlement au travail et le qualifie comme étant contraire à la dignité de la personne, mais elle contient également des dispositions relatives à la rupture du contrat de travail. D'une part, au regard de l'auteur des faits, le paragraphe 1 de l'article 160 du Code du travail, ajoute le comportement de harcèlement au travail comme nouveau motif de licenciement sans droit à une indemnisation. Ainsi, l'employeur peut décider de destituer de son emploi le salarié qui harcèle un autre salarié, dans les termes décrits à l'article 2 du Code du travail. D'autre part, si l'employeur a harcelé le salarié ou que ce dernier a été harcelé par d'autres salariés et que l'employeur n'a pas pris les mesures nécessaires pour protéger la victime, alors, cette dernière peut recourir à la notion de licenciement indirect ou d'autolicenciement. Parallèlement à la mise jeu de l'autolicenciement, il est indiqué que le salarié peut réclamer les autres indemnités auxquelles il a droit.

La réglementation sur le harcèlement au travail n'est pas limitée aux salariés du secteur privé, comme cela a été mentionné plus haut, puisque la loi n 20.607 stipule expressément que la loi $n^{\circ} 18.834$ sur le statut administratif et la loi $n^{\circ} 18.883$ sur le statut administratif des fonctionnaires municipaux sont modifiées pour que la réglementation sur le harcèlement au travail s'applique aux fonctionnaires dans les termes énoncés au deuxième paragraphe de l'article 2 du Code du travail.

Force est de constater que la loi $n^{\circ} 20.607$, ainsi que l'incorporation dans la réglementation de certains des critères appliqués par la jurisprudence en l'absence de réglementation expresse ${ }^{8}$, a permis de renforcer la protection judiciaire en cas de harcèlement au travail.

6 Ibid.

7 Ibid.

8 Par exemple, concernant l'exigence de répétition du comportement du comportement constitutif d'un harcèlement, on peut mentionner l'arrêt de la Cour d'appel de Santiago, Rol n 1517/2011, du 14 décembre 2011, qui affirme que, bien que le juge ait été convaincu que les insultes, les grossièretés et les remarques péjoratives à l'encontre du salarié étaient prouvées, " puisqu'il s'agit d'un seul épisode, cela ne peut être qualifié de harcèlement, car pour qu'il soit reconnu comme tel, d'autres faits du même type doivent être prouvés, ce qui n'a pas été le cas en l'espèce ». 


\section{II - MESURES ADOPTÉES PAR LES TRIBUNAUX DU TRAVAIL EN MATIÈRE DE HARCÈLEMENT AU TRAVAIL}

Nous allons maintenant examiner quelques décisions des tribunaux chiliens qui, dans le cadre de notre législation sur le harcèlement au travail, ont résolu des questions intéressantes pour le développement et la consolidation de la protection des salariés victimes d'un tel comportement.

Concernant le harcèlement au travail d'une salariée qui continuait à fournir ses services à son employeur, la décision de la Cour suprême Rol n 6870/2016 (Recours pour l'unification de la jurisprudence du travail) du 30 novembre 2016 visait à déterminer si le juge du travail, intervenant dans une procédure de protection des droits fondamentaux, était habilité à accorder une indemnisation pour le préjudice moral causé par un tel acte. La Cour s'appuyant sur l'article 495, paragraphe 4, du Code du travail ${ }^{9}$ considéra que "la directive du législateur vise à rétablir un équilibre rompu par la violation des droits fondamentaux, ce qui implique non seulement de mettre fin au comportement préjudiciable, mais donne également au juge de larges pouvoirs pour y parvenir, y compris la réparation des dommages et, en particulier, du préjudice moral ». La Cour ajoute que " même si rien ne peut ramener la victime à l'atmosphère de travail cordiale d'avant, [...] l'indemnisation accordée permet, par équivalence, de soulager l'inconfort, l'angoisse et la souffrance causés par le harcèlement et l'intimidation au travail auxquels elle a été soumise, en rétablissant l'équilibre perdu ». L'arrêt Rol n² 28922/2015 (Unification de la jurisprudence du travail) du 13 juillet 2016, avait statué dans le même sens.

Le jugement du tribunal du travail de Valparaiso, RIT T 245-2016, du 10 février 2017, traite de la situation de ceux qui, après avoir été harcelés par leur employeur, sont finalement licenciés, étant entendu que cette décision de licenciement est l'aboutissement d'un processus de harcèlementet de persécution au travail. Les faits établis par le tribunal du travail étaient les suivants « l'entreprise dénoncée, par l'intermédiaire d'un de ses représentants, a harcelé et a agressé la plaignante sur son lieu de travail, portant ainsi atteinte à son image personnelle et professionnelle au sein de l'organisation, conduisant à une dépression diagnostiquée par un psychiatre et à son licenciement, presque immédiatement après avoir dénoncé ladite situation à l'Inspection du travail, organisme qui aurait également constaté cette agression et fondant ce licenciement sur des paiements frauduleux ou malhonnêtes qui, en somme, n'ont pas été prouvés. La conduite de l'employeur était donc disproportionnée, non motivée, et son pouvoir de commandement a été transféré, ce qui, à son tour, constitue une violation des droits d'une grande signification et importance, assurés et protégés par notre Constitution et par l'ensemble de notre système juridique, comme le droit à l'intégrité psychologique, à l'honneur et à la garantie d'indemnisation... ». II est intéressant de noter que même si le procès vise à dénoncer le licenciement comme une violation des droits fondamentaux, le tribunal prend en compte le harcèlement comme une situation qui durait depuis longtemps, et pas seulement la décision de rompre le contrat, pour conclure qu'il y a eu violation des droits à l'intégrité psychologique, à l'honneur et à la garantie d'indemnisation.

9 Cet article prévoit que « le juge doit veiller à ce que la situation soit ramenée à celle qu'elle était immédiatement avant la survenance de la violation faisant l'objet de la plainte et s'abstient d'autoriser tout type d'accord qui laisserait impuni un comportement qui porte atteinte aux droits fondamentaux ». 
Nous avons déjà indiqué que dans le règlement statutaire de la fonction publique, il existe des règles de fond concernant le harcèlement au travail. Toutefois, lorsque le plaignant est un fonctionnaire régi par un statut spécial, il est pertinent de se référer à la décision de l'organe compétent pour examiner une plainte de harcèlement au travail. La Cour suprême, dans son arrêt Rol n²333/2014 du 20 mai 2015 (Unification de la jurisprudence du travail) énonce clairement que les salariés régis par le statut administratif des fonctionnaires municipaux, loi $n^{\circ} 18.883$, et le statut de l'enseignement, loi $n^{\circ} 19.070$, doivent s'en remettre aux tribunaux du travail pour toute plainte de harcèlement au travail. En effet, bien que la loi n 18.883 fasse référence au harcèlement au travail, on peut affirmer qu'elle «ne prévoit pas de procédure spéciale pour la protection des droits fondamentaux, mais décrit simplement les interdictions auxquelles sont soumis les fonctionnaires municipaux ». Le tribunal rappelle néanmoins que la protection conférée par le Code du travail et sanctionnée par le juge du travail, couvre tout acte survenant dans la relation de travail qui, du fait de l'exercice des pouvoirs de l'employeur, implique une violation des droits fondamentaux du salarié, et qu'elle est applicable dans l'hypothèse du vide juridique d'un statut particulier. Dans le même ordre d'idées, on peut citer l'arrêt de la Cour d'appel de Santiago, Rol n 1096/2015, du 16 septembre $2015^{10}$.

Comme précédemment indiqué, la victime de harcèlement au travail peut avoir recours à la théorie du licenciement indirect ou de l'autolicenciement pour mettre fin à la relation de travail au sein de laquelle elle a été victime de harcèlement. On en trouve un exemple clair dans le jugement du tribunal du travail de Valparaiso, RIT T-166-2013, du 24 janvier 2014, qui a accepté la demande d'autolicenciement d'une salariée pour des actes constitutifs de harcèlement au travail. Le juge considère que la persécution dont a été victime la salariée, sous forme de harcèlement permanent, humiliant et grave de la part de deux managers, constitue une violation du droit du travail par l'employeur, dans la mesure où ils représentaient l'employeur. La Cour d'appel de Valparaiso, saisie d'un recours en annulation ${ }^{11}$, réaffirma, dans son arrêt du 24 avril 2014, que le juge de première instance avait établi l'existence évidente d'intimidations, ce qui constitue du harcèlement moral ou du harcèlement au travail, selon les termes de l'article 2 du Code du travail. En conséquence, l'employeur fut condamné à verser l'indemnité prévue par la loi à la salariée qui avait décidé de mettre fin à son contrat parce qu'elle était victime de harcèlement au travail.

10 L'arrêt indique que « dans le statut administratif, il n'existe pas de règles régissant une procédure juridictionnelle spéciale pour entendre et résoudre les plaintes pour violation des droits fondamentaux des fonctionnaires dans le domaine des relations de travail, la procédure de plainte étant celle de l'article 160 du Statut administratif, auquel le requérant se réfère, et qui n'est qu'un recours administratif devant le Bureau du Contrôleur général de la République. II peut être saisi pour des vices de procédure, dus à des défauts d'un acte administratif, ce qui n'est pas comparable au recours juridictionnel de tout salarié, lorsque, en raison de l'exercice des pouvoirs de l'employeur ou, comme dans ce cas, du supérieur qui a les pouvoirs de direction et de décision, il subit une violation de ses droits fondamentaux ". L'arrêt ajoute que «les autres règles citées par le plaignant n'établissent pas une procédure de protection, mais, au contraire, reconnaissent précisément des garanties, réaffirmant le droit des fonctionnaires de voir leurs garanties fondamentales préservées, établissant à cette fin l'interdiction du harcèlement sexuel et au travail, aux termes de l'article 2, paragraphe 2, du Code du travail, de sorte que pour protéger et rendre effective la pleine protection de ces garanties, et d'autres garanties reconnues par la procédure de tutelle, la procédure prévue à l'article 485 du Code du travail soit applicable».

11 Rol n 74-2014. 
Nous avons fait remarquer qu'avant que le harcèlement au travail ne soit reconnu par le législateur et après son incorporation dans le Code du travail, il a été considéré qu'un tel comportement implique une violation des droits fondamentaux des salariés, de sorte que la procédure de protection devant le juge du travail est un mécanisme de garantie idéal. Cependant, il convient de souligner, comme le fait l'arrêt de la Cour d'appel de Santiago ${ }^{12}$, du 4 novembre 2015, que "l'article 184 du Code du travail, relatif au devoir de protection de l'employeur à l'égard du salarié, ne se limite pas à une obligation d'agir comme le prétend le plaignant, et n'est pas non plus exclu par l'existence de la loi sur la protection récemment créée, mais, comme le déclare la Cour, c'est l'un des mécanismes qui visent la protection générale du salarié ». Dans ce cas, le plaignant n'avait pas agi dans le cadre d'une procédure de protection des droits fondamentaux, mais avait réclamé des dommages-intérêts pour non-respect de l'obligation légale et contractuelle de sécurité prévue aux articles 2, 153 et 184 du Code du travail, dans une relation de travail en cours et à durée indéterminée. La Cour a jugé que «le défendeur a commis des actes assimilables à du harcèlement au travail au sens de l'article 2 du Code du travail, contrevenant ainsi à l'obligation générale de protection du salarié ». La Cour d'appel affirma qu'« en raison de la nature protectrice du droit du travail, les règles doivent être interprétées conformément au principe selon lequel le doute doit profiter au salarié, avec pour conséquence qu'elles doivent bénéficier et non nuire au salarié, comme il résulterait de l'interprétation demandée par le requérant concernant l'existence de deux lois exclusives en matière de protection au travail ». Ainsi, la Cour confirma le point de vue du tribunal de première instance selon lequel « le législateur a mis à la disposition des salariés une série de mécanismes qui visent à assurer leur protection générale, aussi bien physique que psychologique, ainsi que leurs droits fondamentaux, en se référant aux divers statuts de protection que l'on retrouve dans les différentes règles du Code du travail, qu'il cite, concluant ainsi que le statut protecteur est large et non exclusif, reconnaissant que la salariée disposait d'une série de mécanismes, tous aussi valables, pour agir à l'égard de son employeur ».

\section{Pour conclure}

L'entrée en vigueur de la protection spécifique au harcèlement au travail dans le système juridique chilien est le résultat d'un processus en évolution dans lequel la jurisprudence a joué un rôle central. En l'absence d'un texte juridique explicite, c'est la jurisprudence qui a ouvert la voie à la protection en cas de harcèlement au travail. D'autre part, lorsque la loi $n^{\circ} 20.607$ est entrée en vigueur, la délimitation de la zone à protéger a pu être plus précise, et ce, grâce à une définition et un champ d'action qui sont maintenant mieux établis.

Comme nous l'avons vu, la jurisprudence continue de jouer un rôle très important, non pas pour décider si le harcèlement au travail fait l'objet d'une protection ou non, mais plutôt pour définir les mesures réparatrices ou palliatives adoptées. Parmi elles figurent un large éventail de possibilités, allant de la réparation du préjudice moral à la protection des fonctionnaires par le biais de la procédure prévue par le Code du travail.

12 Rol n 948/2015. 
On constate que la grande majorité des cas qui font l'objet de poursuites pour harcèlement impliquent une rupture de la relation de travail. Bien qu'il soit possible $d^{\prime}$ intenter une action en justice et d'obtenir une protection tout en maintenant la relation de travail, la pratique montre que cela est peu fréquent, voire évité.

Le juge du travail au Chili dispose d'un large champ d'action dans le domaine des mesures de réparation. En ce sens, le juge peut aussi bien imposer des formations pour améliorer l'environnement de travail ou simplement exiger des changements au sein de la structure interne de l'entreprise. Il est donc possible d'envisager que ce pouvoir permettra de réaliser de nouveaux progrès dans la protection en cas de harcèlement au travail, par le biais de la jurisprudence. 